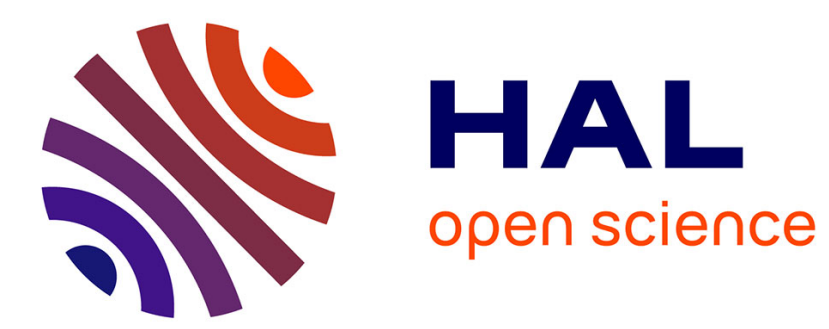

\title{
Nonuniqueness and stability in problems of equilibrium of elastic two-phase bodies
}

\author{
Victor A. Eremeev, Alexander B. Freidin, Leah L. Sharipova
}

\section{To cite this version:}

Victor A. Eremeev, Alexander B. Freidin, Leah L. Sharipova. Nonuniqueness and stability in problems of equilibrium of elastic two-phase bodies. Doklady Physics, 2003, 48 (7), pp.359-363. hal-00826484

\section{HAL Id: hal-00826484 \\ https://hal.science/hal-00826484}

Submitted on 4 Jun 2013

HAL is a multi-disciplinary open access archive for the deposit and dissemination of scientific research documents, whether they are published or not. The documents may come from teaching and research institutions in France or abroad, or from public or private research centers.
L'archive ouverte pluridisciplinaire HAL, est destinée au dépôt et à la diffusion de documents scientifiques de niveau recherche, publiés ou non, émanant des établissements d'enseignement et de recherche français ou étrangers, des laboratoires publics ou privés. 


\title{
Nonuniqueness and Stability in Problems of Equilibrium of Elastic Two-Phase Bodies
}

\author{
V. A. Eremeev*, A. B. Frĕ́din**, and L. L. Sharipova** \\ Presented by Academician N.F. Morozov March 3, 2003
}

Interfaces in martensitic transformations can be treated as strain-discontinuity surfaces, where, in addition to the ordinary kinematic and force conditions, an additional thermodynamic condition must be satisfied [1-4]. This condition restricts the geometry of the interfaces [5-8].

Being a problem with an unknown boundary, the problem of determination of equilibrium two-phase configurations has as a rule several solutions; i.e., different equilibrium two-phase structures can correspond to the same boundary conditions. In this case, locally stable (metastable) states, as well as states providing the global minimum of the energy functional, are of interest. A certain state is realized depending on the initial conditions and kinetics of a phase transition. However, various equilibrium solutions can be a priori estimated disregarding transition kinetics by analyzing the stability of two-phase fields of strains and energy changes induced by phase transitions.

In this paper, the nonuniqueness and stability of two-phase states are analyzed by an example of centrosymmetric two-phase strain fields. The equilibrium of a two-phase ball on the class of solutions with one spherical interface was previously analyzed in [6-9]. In these works, the possibility of two or more equilibrium interfaces, as well as the stability of two-phase states under arbitrary disturbances, was not studied. The results presented below continue investigations begun in $[10,11]$.

\section{EQUILIBRIUM CENTROSYMMETRIC STRAINS}

For small strains, the problem of equilibrium twophase configurations of an elastic body reduces $[7,12]$

\footnotetext{
* Rostov State University.

ul. Zorge 5, Rostov-on-Don, 344090 Russia

e-mail:eremeyev@math.rsu.su

**Institute of Problems of Mechanical Engineering, Russian Academy of Sciences, Vasil'evskiu Ostrov, Bol'shoi pr. 61, St. Petersburg, 199178 Russia e-mail:freidlin@mechanics.ipme.ru
}

to the problem of determination of an interface $\Gamma$ and displacement field $\mathbf{u}(x)$, which is quite smooth for $x \notin \Gamma$, is continuous on $\Gamma$, and satisfies the boundary conditions and equilibrium conditions

$$
\begin{gathered}
x \notin \Gamma: \quad \nabla \cdot \sigma=0, \quad \theta=\text { const, } \\
x \in \Gamma: \quad[\mathbf{u}]=0, \quad[\sigma] \cdot \mathbf{n}=0, \\
{[f]-\sigma:[\varepsilon]=0 .}
\end{gathered}
$$

Here, $x$ is a point of the body; the volume density $f$ of the free energy is approximated by the quadratic functions

$$
\begin{gathered}
f(\varepsilon, \theta)=\min _{-,+}\left\{f^{-}(\varepsilon, \theta), f^{+}(\varepsilon, \theta)\right\}, \\
f^{ \pm}(\varepsilon, \theta)=f_{0}^{ \pm}(\theta)+\frac{1}{2}\left(\varepsilon-\varepsilon_{ \pm}^{p}\right): C_{ \pm}:\left(\varepsilon-\varepsilon_{ \pm}^{p}\right) .
\end{gathered}
$$

$\varepsilon$ and $\sigma$ are the strain tensor and stress tensor, respectively; $\theta$ is the temperature; $\mathbf{n}$ is the unit vector normal to the interface $\Gamma ; C_{ \pm}$are the positive definite tensors of the elastic moduli of the phases; $f_{0}^{ \pm}$and $\varepsilon_{ \pm}^{p}$ are, respectively, the free-energy densities and strain tensors for the phases in unstressed state; $[\cdot]=(\cdot)_{+}-(\cdot)_{-}$; and subscripts + and - mean the material in different phase states. Mass forces, thermoelastic stresses, and surface energy are ignored.

Thermodynamic condition (2) reduces to an equation determining a one-parameter family of normals to the interface depending on strains on the one side of the interface [5]. If the tensor $C_{1}=C_{+}-C_{-}$is nondegenerate, this equation is represented in the $q$ space as

$$
\begin{gathered}
\chi\left(q_{ \pm}, \mathbf{n}\right) \\
\triangleq \gamma_{*}+\frac{1}{2}\left(q_{ \pm}: C_{1}^{-1}: q_{ \pm} \pm q_{ \pm}: K_{\mp}(\mathbf{n}): q_{ \pm}\right)=0, \\
q=-C_{1}: \varepsilon+\left[C: \varepsilon^{p}\right], \\
K_{ \pm}(\mathbf{n})=\left\{\mathbf{n} \otimes G_{ \pm} \otimes \mathbf{n}\right\}^{s}, \quad G_{ \pm}=\left(\mathbf{n} \cdot C_{ \pm} \cdot \mathbf{n}\right)^{-1}, \\
\gamma_{*}=\gamma+\frac{1}{2}\left[\mathbf{e}^{p}\right]: B_{1}^{-1}:\left[\mathbf{e}^{p}\right], \quad B_{ \pm}=C_{ \pm}^{-1}, \\
B_{1}=B_{+}-B_{-},
\end{gathered}
$$




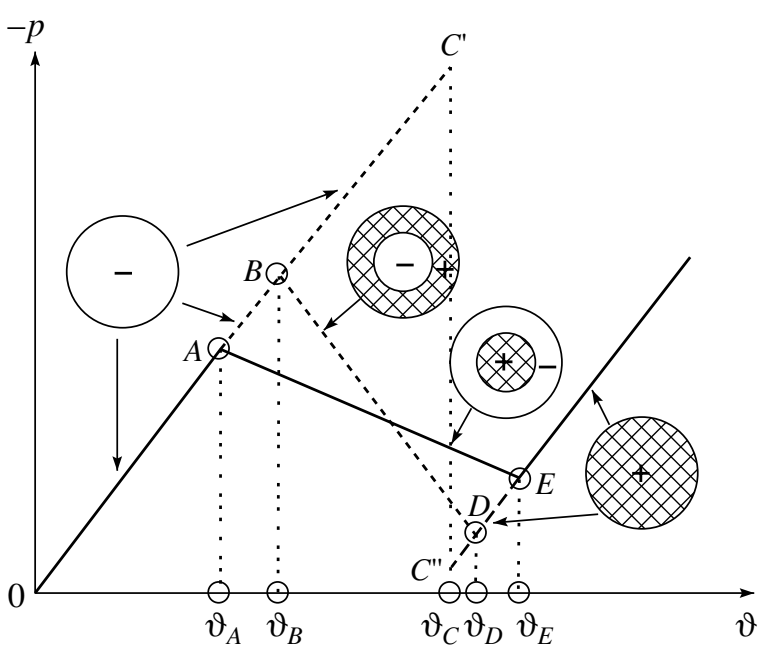

Fig. 1. Pressure vs. volume strain for $\mu_{+}>\mu_{-}$, where $\mu_{+}$and $\mu_{-}$are the shear moduli of the respective phases.

where the superscript $s$ means symmetrization: $K_{i j k l}=$ $n_{(i} G_{j)(k} n_{l)}$.

The following statement for an isotropic material can be proved by analyzing the possibility of satisfaction of equilibrium condition (4) at several interfaces.

Statement. For the class of centrosymmetric equilibrium two-phase solutions, only one spherical interface for a solid body and no more than two interfaces in a body with a spherical cavity can exist.

This statement is valid for any type of boundary conditions on the cavity surface.

\section{STABILITY. \\ THE LINEARIZED BOUNDARY VALUE PROBLEM FOR A TWO-PHASE BODY}

We consider a certain initial equilibrium two-phase state and a state infinitesimally different from it. The displacement and radius vector of the interface in the perturbed state are given by the formulas

$$
\mathbf{u}=\mathbf{u}^{0}+\mathbf{w}, \quad \mathbf{r}=\mathbf{r}^{0}+\eta \mathbf{n},
$$

where $\mathbf{w}$ is the vector of small additional displacements, $\eta$ is the perturbation of the interface in the $\mathbf{n}$ direction, and $\mathbf{u}^{0}$ and $\mathbf{r}^{0}$ are the displacement vector and radius vector of the interface in the initial state, respectively. We consider quasistatic perturbations; i.e., the field of displacements depends on time as on a parameter through the time dependence of the interface.

When the interface moves quasistatically, $\chi \neq 0$ is the thermodynamic force. In the linear thermodynamic approximation, the normal component of the interface velocity is determined by the equation

$$
v_{n}^{\Gamma}=-k \chi\left(q_{+}, \mathbf{n}\right), \quad k>0,
$$

where the normal is out of the domain occupied by the + phase and $k$ is the kinetic coefficient.

The linearization of the boundary value problem specified by Eqs. (1) and (2) provides the set of equations for $\mathbf{w}$ and $\eta$

$$
\begin{gathered}
x \notin \Gamma: \quad \nabla \cdot \sigma_{ \pm}(\mathbf{w})=0, \quad \sigma_{ \pm}(\mathbf{w})=C_{ \pm}: \nabla \mathbf{w}, \\
x \in \Omega_{1} \cup \Omega_{2}:\left.\quad \mathbf{w}\right|_{\Omega_{1}}=0,\left.\quad \mathbf{n} \cdot \sigma(\mathbf{w})\right|_{\Omega_{2}}=0, \\
x \in \Gamma: \quad[\mathbf{w}]=-\eta\left[\mathbf{n} \cdot \nabla \mathbf{u}^{0}\right], \\
\mathbf{n} \cdot[\sigma(\mathbf{w})]=\nabla \eta \cdot\left[\sigma^{0}\left(\mathbf{u}^{0}\right)\right]-\eta \mathbf{n} \cdot\left[\mathbf{n} \cdot \nabla \sigma^{0}\left(\mathbf{u}^{0}\right)\right], \\
q_{+}:\left(C_{1}^{-1}+K_{-}(\mathbf{n})\right): q_{+}+\eta \mathbf{n} \cdot \nabla \chi=-\frac{1}{k} \frac{d \eta}{d t}, \\
q_{+} \triangleq-C_{1}: \varepsilon(\mathbf{w}),
\end{gathered}
$$

where $\Omega_{1} \cup \Omega_{2}$ is the outer boundary of the body. Relation (10) follows from master equation (7) and describes the evolution of initial perturbations of the interface. If the initial state includes several interfaces, consistency conditions (9) and (10) are imposed at each of them.

The operator generated by the boundary value problem specified by Eqs. (8) and (9) is uniquely solvable with respect to $\mathbf{w}$, because additional displacements $\mathbf{w}$ are expressed in terms of the interface perturbation $\eta$ as $\mathbf{w}=\mathscr{A} \eta$, where $\mathscr{A}$ is a certain linear integral operator. The substitution of this expression into master equation (10) provides the integro-differential equation for $\eta$

$$
-\frac{1}{k} \frac{d \eta}{d t}=\mathscr{L} \eta
$$

For several interfaces, the solution of Eqs. (8)-(10) leads to the set of integro-differential equations for perturbations of the interfaces.

The analysis of the stability of equilibrium solutions reduces to the determination of the bifurcation points of Eq. (11) and behavior of its small solutions. Bifurcation points are determined from the existence condition of stationary nonzero solution of the equation $\mathscr{L} \eta=0$. If the operator $\mathscr{L}$ is positive definite, Eq. (11) (set of equations) admits only solutions decreasing with time. In this case, the initial two-phase solution is stable. Otherwise, undamped perturbations exist and, therefore, the solution is unstable. 


\section{STABILITY}

\section{OF CENTROSYMMETRIC EQUILIBRIUM} STATES

Figure $1[6,7]$ shows pressure $p$ on the surface of a solid ball as a function of $\vartheta=\frac{3 u_{0}}{R}$, where $u_{0}$ are radial displacements at the ball boundary and $R$ is the outer radius of the ball.

The solution changes qualitatively at the points $A, B$, $D$, and $E$, where the topology of the solution changes and an internal interface can appear. Single-phase solutions presented by intervals $A C^{\prime}$ and $C^{\prime \prime} E$ in Fig. 1 are locally stable on the class of one-phase deformations. At the same time, both two-phase solutions presented by intervals $A E$ and $B D$ are energetically favorable over the single-phase solution and are stable on the class of centrosymmetric solutions, where only the interface radius is perturbed for given $\vartheta$ values [7].

Analysis of energy changes accompanying the appearance of new-phase domains shows that centrosymmetric two-phase states energetically favorable over one-phase states even disregarding the thermodynamic equilibrium condition first appear when achieving $\vartheta_{A}$ (loading) and $\vartheta_{B}$ (discharge). This means that the single-phase solution is metastable with respect to two-phase solutions, i.e., is unstable with respect to finite two-phase perturbations. Therefore, the points $\vartheta_{A}$ and $\vartheta_{B}$ can be called points of topological instability (bifurcation).

For a body with a spherical cavity, similar dependences can be plotted for equations with one and two interfaces and for various phase alternating. For twophase solutions, the new-phase domain expands and pressure decreases with an increase in $\vartheta$.

We consider the stability of the above solutions with respect to axisymmetric perturbations.

Equilibrium conditions for the solid ball admit two two-phase solutions. It is shown that a solution where the phase with the higher shear modulus occupies the outer spherical layer is unstable (interval $B D$ in Fig. 1) $[10,11]$. When the harder phase is located at the center of the ball, loss of stability is not observed (interval $A E)$. Solid lines in Fig. 1 are solutions for the solid ball that are locally stable and energetically favorable. Dashed lines are unstable or metastable solutions.

Equilibrium conditions for the ball with the cavity admit solutions with one and two interfaces and various phase alternating. The analysis of stability, when the bulk moduli of materials of phases are identical, for zero stresses or displacements on the cavity surface provides the following conclusions.

(i) When the cavity is small, only a solution with one interface, where the phase with the higher shear modu- lus forms the inner layer, can be stable. Other solutions, including those with two interfaces, are unstable.

(ii) When the radius of the cavity exceeds a certain value, all two-phase solutions are unstable.

Comparison of results for the stability of the solid ball and ball with the cavity reveals the passage to the limit: the stable solution for the ball with the cavity passes to the stable solution for the solid ball when the cavity radius tends to zero.

\section{TWO-PHASE STRAIN FIELDS AND PHASE-TRANSITION ZONE}

We compare the strain fields in various two-phase configurations of the ball with the phase-transition zone formed in the strain space by all strains that can coexist at the equilibrium interface [8, 12-14].

For centrosymmetric two-phase fields, spherical and axisymmetric fields of the $q$ tensor arise in the body. In the principal-value space $q_{1}, q_{2}$, and $q_{3}$ of the $q$ tensor, these fields lie on the $q_{2}=q_{3}$ plane. Figure 2 shows the sections of the phase-transition zone by this plane. The two solutions of the problem for the solid ball correspond to jumps $a a^{\prime}$ and $b b^{\prime}$ at the interface.

For $\mu_{1}>0$, the $A E$ solution (Fig. 1), where the inner + phase has higher shear modulus, corresponds to the strain distribution shown in Fig. 2a by the point $a$ (+ phase in the hydrostatic state) and interval $a^{\prime} e$ (strains in the ball layer formed by the - phase). When the point $e$ in the $z_{0} e$ path is achieved, the + phase can originate at the center of the ball and an interface with the strain jump $a a^{\prime}$ can arise. The interface expands with further deformation of the ball. The strain of the inner + phase remains unchanged (point $a$ ). Strains in the ball layer of the - phase are represented by the points of the interval $a^{\prime} e$, but the point $e$ is shifted to $a^{\prime}$ with deformation.

The second solution ( $B D$ in Fig. 1) is represented in Fig. $2 \mathrm{a}$ by the jump $b b^{\prime}$ and interval $b^{\prime} d$.

For $\mu_{1}<0$, as the point $a$ is achieved, the + phase originates on the ball surface with the strain $a^{\prime}$ at the interface (Fig. 2b). With the transformation of the ball, strains in the ball layer of the + phase are distributed over the interval $a^{\prime} e$. The second solution is represented in Fig. $2 \mathrm{~b}$ by the jump $b b^{\prime}$ and interval $b^{\prime} d$.

Thus, various two-phase states of the ball can correspond to strain fields of the following two types.

(i) Strains at the interface correspond to the outer boundary of the phase-transition zone, and strains at other points of the body lie beyond the phase-transition zone (interval a'e in Fig. 2).

(ii) At least in a part of the body, strains correspond to inner domains of the phase-transition zone (interval $d b^{\prime}$ ), and a jump occurs form the nonconvex part of the boundary of the phase-transition zone (point $b$ ). 


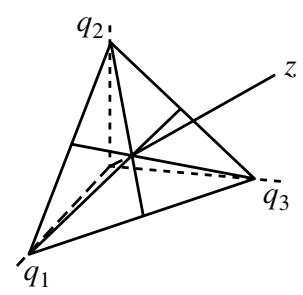

(a)
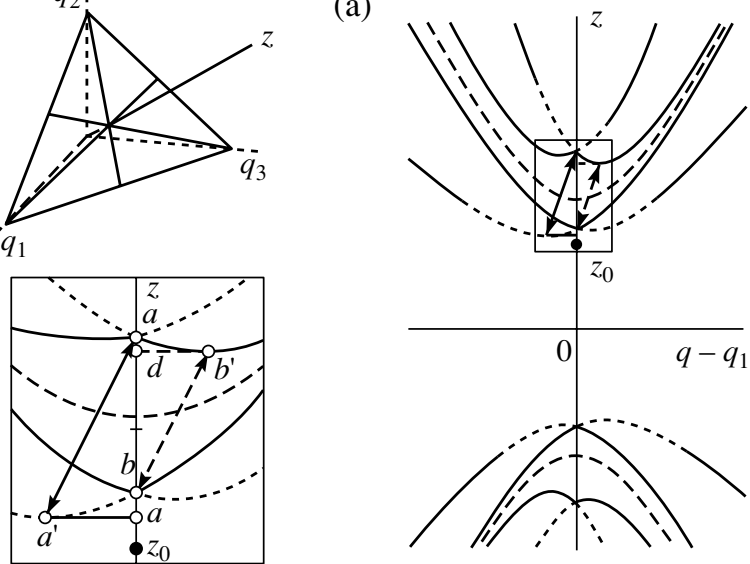

(b)

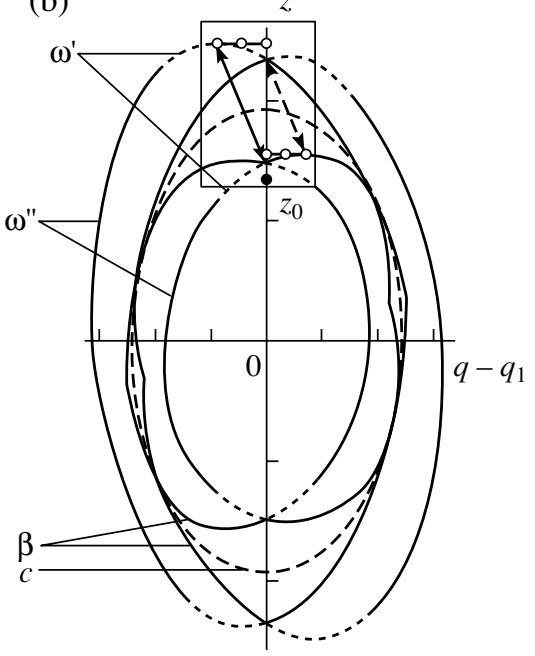

Fig. 2. Section of the phase-transition zone by the $q_{2}=q_{3}=$ $q$ plane for (a) $\mu_{1}>0$ and $K_{1}<0$ and (b) $\mu_{1}<0$ and $K_{1}<0$ : $\omega^{\prime}$ and $\omega^{\prime \prime}$ are the outer boundaries of the zone, $\beta$ are the inner boundaries, $c$ is the surface, where the derivatives of the function $f(\varepsilon)$ are discontinuous, $z=\frac{I_{1}(q)}{\sqrt{3}}$, and $z_{0}$ is the initial undeformed state.

In the case considered above, the unstable solution corresponds to the second type of two-phase strains.

\section{CONCLUSIONS}

The above analysis reveals a number of fundamental features of the formulation and solution of boundary value problems for bodies that undergo phase transitions under deformation and, therefore, have an additional degree of freedom associated with an interface.

(i) Since the boundary value problem specified by Eqs. (1) and (2) is nonlinear due to the presence of an unknown interface, the solution is not unique.

(ii) The new degree of freedom significantly affects the stability of elastic two-phase bodies. In the absence of a phase transition $(\eta=0)$, the problem specified by
Eqs. (8) and (9) is a well-known homogeneous boundary value problem for a composite, linearly elastic body and has only zero solutions. In this case, the centrosymmetric strain field is stable for any radius of the fixed interface.

(iii) The appearance of a cavity, i.e., an additional outer boundary, is responsible for a destabilizing action independent of the type of boundary conditions imposed at it.

(iv) Solutions of the problem of equilibrium twophase configurations of elastic bodies can substantially differ from solutions obtained when designing twophase composites of the optimal structure. Centrosymmetric solutions providing the global minimum of the energy functional of the two-phase body for a fixed fraction of one of the phases were considered in [15]. The phases differed only in the elastic moduli; i.e., this case corresponds to $\varepsilon^{p}=0$. A solution providing the global minimum for the solid ball was shown to correspond to a structure where the harder phase is concentrated at the center of the ball and is surrounded by the softer phase. This conclusion coincides with the results of the above stability analysis.

However, for the ball with the cavity, when the bulk moduli of materials of the phases are identical, a solution in the form of the three-layered shell, whose middle layer is formed by the hard phase, is optimal (energetically favorable on the class of states satisfying the isoperimetric condition of the constant content). As was shown above, this solution is unstable under phase transitions, which mean the appearance of the additional degree of freedom associated with the change in the relative phase content.

\section{ACKNOWLEDGMENTS}

This work was supported by the Ministry of Industry, Science, and Technology of the Russian Federation (project no. 40.010.1 1.1195), the Russian Foundation for Basic Research (project nos. 01-01-00324, 02-01-06263), and the Competitive Center for Fundamental Natural Sciences at St. Petersburg State University (project no. E00-4.0-185).

\section{REFERENCES}

1. M. A. Grinfel'd, Methods of Continuum Mechanics in the Theory of Phase Transitions (Nauka, Moscow, 1990).

2. R. D. James, Arch. Ration. Mech. Anal. 77, 143 (1981).

3. M. E. Gurtin, Arch. Ration. Mech. Anal. 84, 1 (1983).

4. V. G. Osmolovski1̌, Variational Problem of Phase Transitions in Continuum Mechanics (St.-Peterburg. Gos. Univ., St. Petersburg, 2000).

5. L. B. Kublanov and A. B. Frĕ̌din, Prikl. Mat. Mekh. 52 (3), 493 (1988). 
6. N. F. Morozov, I. R. Nazyrov, and A. B. Frel̆din, Dokl. Akad. Nauk 346, 188 (1996) [Phys. Dokl. 41, 40 (1996)].

7. I. R. Nazyrov and A. B. Frě̆din, Izv. Akad. Nauk, Mekh. Tverd. Tela 5, 52 (1998).

8. N. F. Morozov and A. B. Freĭdin, Tr. Mat. Inst., Ross. Akad. Nauk 223, 220 (1998).

9. V. A. Eremeev and L. M. Zubov, Izv. Akad. Nauk, Mekh. Tverd. Tela 2, 56 (1991).

10. L. L. Sharipova, V. A. Eremeev, and A. B. Frĕ̌din, Izv. Vyssh. Uchebn. Zaved., Sev.-Kavk. Reg., Estestv. Nauki, Special Issue: Mathematical Simulation and Numerical Experiments in Mechanics and Physics (2001), pp. 166-169.
11. V. A. Eremeev, A. B. Frel̆din, and L. L. Sharipova, Problems of Mechanics of Deformable Body (St.-Peterburg. Gos. Univ., St. Petersburg, 2002), pp. 111-122.

12. A. B. Frě̆din, Issled. Uprugosti Plast. 18, 266 (1999).

13. A. B. Frĕdin and A. M. Chiskis, Izv. Akad. Nauk, Mekh. Tverd. Tela 29 (4), 91 (1994).

14. A. B. Freidin, E. N. Vilchevskaya, and L. L. Sharipova, Theor. Appl. Mech. 28/29, 149 (2002).

15. R. Fosdick and G. F. Royer-Carfagni, J. Elast. 42, 49 (1996). 